\title{
Theory of the composition dependence of the band offset and sheet carrier density in the $\mathrm{GaN} / \mathrm{Al}_{x} \mathrm{Ga}_{1-x} \mathrm{~N}$ heterostructure
}

\author{
S. Satpathy \\ Department of Physics, University of Missouri, Columbia, Missouri 65211 and Air Force Research \\ Laboratory, Materials and Manufacturing Directorate, Wright-Patterson Air Force Base, Ohio 45433 \\ Z. S. Popovic a) \\ Department of Physics, University of Missouri, Columbia, Missouri 65211 \\ W. C. Mitchel \\ Air Force Research Laboratory, Materials and Manufacturing Directorate, Wright-Patterson \\ Air Force Base, Ohio 45433
}

(Received 8 December 2003; accepted 20 February 2004)

\begin{abstract}
We present a systematic study of the sheet carrier density and valence-band offset in the $\mathrm{GaN} / \mathrm{Al}_{x} \mathrm{Ga}_{1-x} \mathrm{~N}(0001)$ heterostructure as a function of $x$ from $a b$ initio density-functional methods. We find that the calculated sheet carrier density increases rapidly with $x$ for $x \leqslant 0.3$ in good agreement with experiments, but beyond this concentration, it quickly saturates to a value of about $2 \times 10^{13} \mathrm{~cm}^{-2}$. The band offset shows a small asymmetry between the Ga-face and $\mathrm{N}$-face interfaces and changes more or less linearly with $x$, although a small bowing is found. The layer-projected densities of states indicate the formation of the two-dimensional electron gas at the Ga-face interface and confirm the absence of interface states in the gap. (C) 2004 American Institute of Physics.
\end{abstract}

[DOI: $10.1063 / 1.1704869$ ]

\section{INTRODUCTION}

Nitride-based quantum well structures show great promise for optoelectronic device applications. A key property of the III-V nitrides, different from traditional semiconductors, is the presence of a large spontaneous polarization, arising out of a lack of inversion symmetry of the wurtzite crystal structure. Thus, for the nitride heterostructures grown along a polar axis, the polarization difference between the two materials produces a sheet charge density at the interface, which in turn is compensated by a screening charge of opposite sign. For the $\mathrm{GaN} / \mathrm{Al}_{x} \mathrm{Ga}_{1-x} \mathrm{~N}$ grown along [0001], a highly mobile two-dimensional electron gas (2DEG) is formed. The charge in the $2 \mathrm{DEG}$ can be modified via the piezoelectric effect of the cap layer, and densities in the range of $10^{13} \mathrm{~cm}^{-2}$ may be reached. Such a large carrier density combined with high mobility leads to good conductivity and superior device performance.

To manipulate the electrical and optical properties of these devices effectively, an accurate determination of the sheet charge density as well as of the band offset is necessary. Knowledge of the latter is vital for assessing the degree of carrier confinement and therefore the usefulness of the material for device applications. The band offset has been studied for mostly the end member GaN/AlN, although experimental work to determine the variation of the offset with Al concentration has just begun. ${ }^{1}$ Concerning the sheet charge density, there are several measurements reported in the literature, with most of the theoretical works based on the macroscopic polarization theory. In this paper, we study the

\footnotetext{
a) Permanent address: Institute for Nuclear Sciences-"Vinca", PO Box: 522,
} 11001 Belgrade, Yugoslavia. variation of the band offset and the sheet carrier density in the $\mathrm{GaN} / \mathrm{AlGaN}$ heterostructure as a function of the $\mathrm{Al}$ concentration from $a b$ initio density-functional theory (DFT).

\section{METHOD OF CALCULATION}

The calculations reported here are based on the local density approximation to the density-functional theory. The linear muffin-tin orbitals method ${ }^{2}$ in the atomic spheres approximation (LMTO-ASA) was used to solve the KohnSham equations self-consistently. In addition to the atoms, an equal number of empty spheres were included to better describe the charge density in the interstitial region. Spin-orbit coupling was not included, being negligible for the atoms involved, and also we treated the Ga $3 d$ electrons as core electrons.

We used a supercell technique, with the unit cell consisting of either $(\mathrm{GaN})_{n} /(\mathrm{AlN})_{n}$ or $\left(\mathrm{Ga}_{4} \mathrm{~N}_{4}\right)_{n} /\left(X_{4} \mathrm{~N}_{4}\right)_{n}$ layers periodically repeated along [0001] (64 atoms plus equal number of empty spheres in the second case with $n=4$ ). Individual $X$ atoms in the unit cell were either $\mathrm{Ga}$ or $\mathrm{Al}$, allowing us to model the $\mathrm{Al}_{x} \mathrm{Ga}_{1-x} \mathrm{~N}$ layer with varying $\mathrm{Al}$ concentration. There are two types of interface. The "Gaface" interface is encountered going along [0001] from GaN to AlGaN-i.e., along the Ga-to-N bond, and it is called so because a $\mathrm{Ga}$ - (or $\mathrm{Al}-$ ) terminated surface would occur at the $\mathrm{AlGaN} /$ vacuum interface if the structure is grown this way. The "N-face" occurs going in the reverse direction-i.e.,

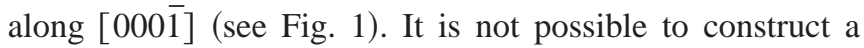
supercell with only one type of interface and our supercell contained one of each of the two interface types. It is the Ga-face interface that is of experimental interest, since it is there that the $2 \mathrm{DEG}$ forms. 


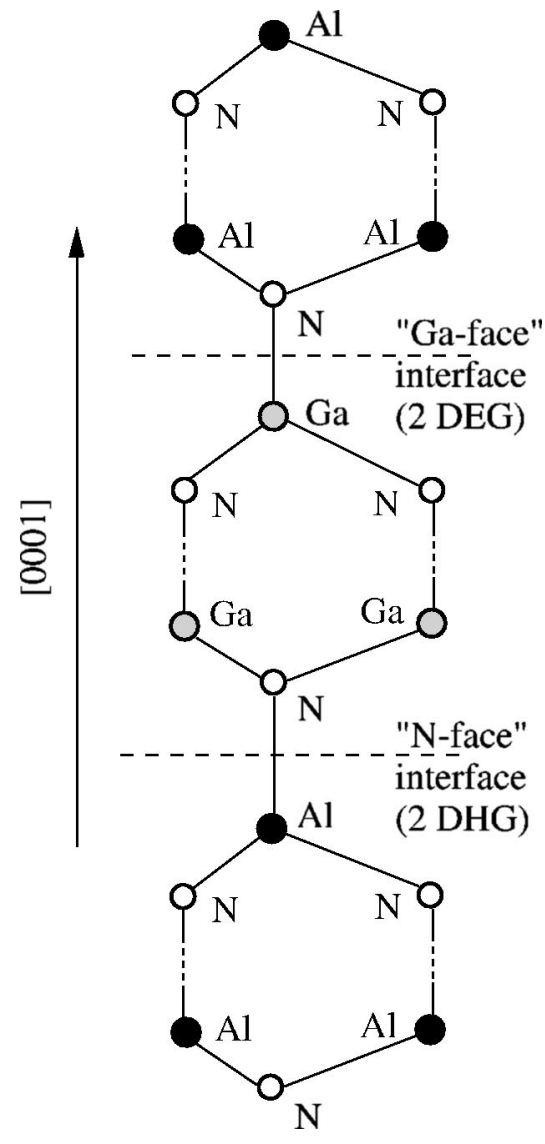

FIG. 1. Sketch of the nitride heterostructure illustrating the two types of interface.

All our calculations were performed for the wurtzite GaN/AlGaN (0001) structure lattice matched to GaN. The common in-plane lattice constant was taken to be the bulk value for $\mathrm{GaN}$. The perpendicular lattice constant $c$ for the AlN epilayer was computed from the macroscopic elasticity theory, ${ }^{3}$ according to which

$$
\left(c-c_{0}\right) / c_{0}=\left(-2 c_{13} / c_{33}\right)\left(a-a_{0}\right) / a_{0},
$$

where $a_{0}$ and $c_{0}$ are the lattice constants for bulk AlN and $a$ and $c$ are the lattice constants for the AlN epilayer. Table I lists the structural parameters used in the calculation. With these, the perpendicular lattice constant for the AlN epilayer is $c=4.911 \AA$, which represents a compression of about $1.4 \%$ from the bulk AlN value. For $\mathrm{Al}_{x} \mathrm{Ga}_{1-x} \mathrm{~N}$, the perpendicular lattice constant was obtained from a linear interpolation between unstrained $\mathrm{GaN}(x=0)$ and strained AlN $(x$ $=1$ ). The internal structure parameter $u$ was taken to be 0.376 for both $\mathrm{GaN}$ and AlN, neglecting the slight difference between the two materials. Earlier total energy DFT calculations for GaN/AlN have indicated that the macroscopic elas-

TABLE I. Lattice parameters and elastic constants for bulk GaN and AlN.

\begin{tabular}{lccc}
\hline \hline \multicolumn{1}{c}{ Material } & $a$ & $c$ & $u$ \\
\hline GaN & $3.189 \AA$ & $5.185 \AA$ & 0.376 \\
AlN & $3.112 \AA$ & $4.982 \AA$ & 0.38 \\
Elastic constants for AlN & $c_{13}=108 \mathrm{GPa}$ & $c_{33}=373 \mathrm{GPa}$ & \\
\hline \hline
\end{tabular}

ticity theory predicts the perpendicular lattice constant for the epilayers quite well and the residual relaxation of the atoms at the interface is negligible. ${ }^{4}$

To compute the valence band offset, we follow several steps: (i) calculation of the electronic structure for the two bulk materials and identification of some reference level in the bulk, which is a local characteristic of the periodic potential seen by the electron, (ii) calculation of the same for the interface using the supercell technique, and (iii) determination of the band offset from the relative position of the reference level on the right and left sides of the interface.

Any convenient characteristic of the potential may be used as the reference level. Following Lambrecht et al., ${ }^{5}$ we use the cell-averaged point-charge Coulomb potential as the reference level, a quantity that is especially easy to calculate in the LMTO-ASA method. The cell-averaged potential $\bar{V}$ may be calculated by first averaging parallel to a plane (planar-averaged potential) and then averaging over a period normal to the plane. We can, alternatively, calculate it by first averaging over the volume of the Wigner-Seitz atomic spheres,

$$
V_{i}=\frac{3 q_{i}}{2 s_{i}}+\sum_{j}{ }^{\prime} \frac{q_{j}}{\left|r_{i}-r_{j}\right|},
$$

and then by averaging over the spheres with a weight factor proportional to their volumes:

$$
\bar{V}=\sum_{i} \Omega_{i} V_{i} / \sum_{i} \Omega_{i},
$$

where $\Omega_{i}=4 \pi s_{i}^{3} / 3$ is the sphere volume, $r_{i}$ is the atomic sphere position, $s_{i}$ is the sphere radius, and $q_{i}$ is the total charge, nuclear plus electronic, for the $i$ th sphere. In Eq. (2), the first term is the average of the point charge $q_{i}$ located at the center of the sphere and the second term is the Madelung potential from all other spheres. For the present case, we compute $\bar{V}$ by averaging over the bilayer atoms and empty spheres (there are two empty spheres in the wurtzite unit cell on either side of each bilayer; we give them $50 \%$ weightage each). Bilayer total charge $Q$ is defined as sum of charges of two atoms in the bilayer plus those of the empty spheres.

The valence-band offset (VBO) may then be calculated by adding the two contributions

$$
\mathrm{VBO}=\Delta \bar{V}+\Delta E_{B S},
$$

where $\Delta \bar{V}$ is the difference in the cell-averaged electrostatic potential across the interface and $\Delta E_{B S}$ is the valence-top difference of the bulk band structure of the two materials, measured with respect to a common cell-averaged potential $\bar{V}$. In our calculation, the valence top occurs $0.34 \mathrm{eV}$ and $0.84 \mathrm{eV}$ above $\bar{V}$, for GaN and strained AlN, respectively. The conduction band offset (CBO) is calculated by adding the gap difference $\Delta E_{g}$ between the two materials forming the interface, taking the strain dependence of the gaps into account. Thus, we have

$$
\mathrm{CBO}=\mathrm{VBO}+\Delta E_{g} .
$$




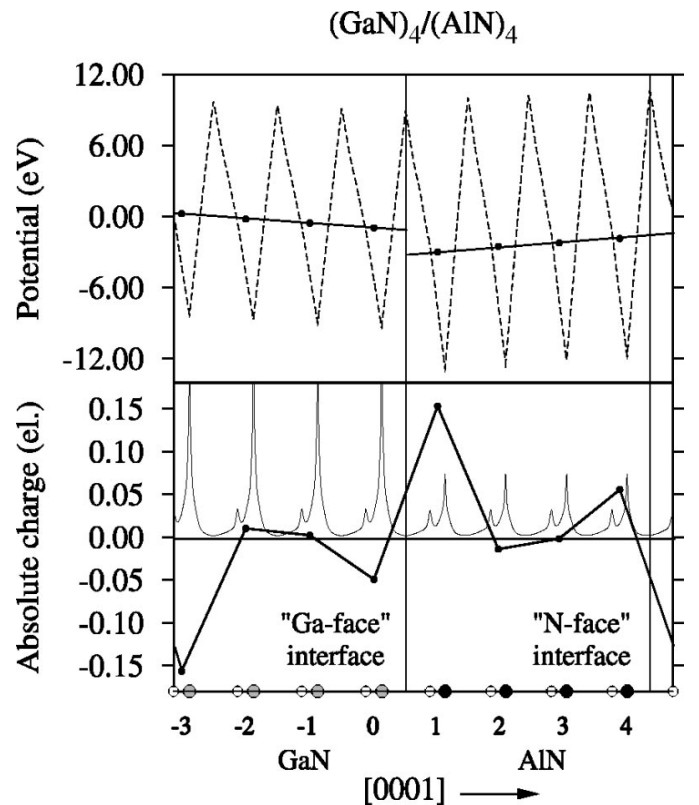

FIG. 2. Planar-averaged (dashed line) and cell-averaged (circles) electrostatic potentials seen by an electron near the interface (upper panel). Straight lines in the upper panel are fits to the data points to compute the electric fields. Lower panel shows the planar-averaged electronic charge as well as the total bilayer charge $Q$ (units of $|e|$ ). While the interface dipole leading to the band offset is clearly noticeable in the lower panel, the monopole charge is best inferred from the change in the electric field across the interface, as seen in the upper panel.

\section{RESULTS AND DISCUSSION}

Figure 2 shows for the GaN/AIN structure the calculated cell-averaged potential and the bilayer total charge, showing the macroscopic electric field as well as the interfacial sheet charge density. The monopole charge produces the change in the macroscopic electric field across the interface, while the dipole moment produces a discontinuity in the potential itself, leading to the band offset. However, with a monopole charge present, the dipole moment is ill defined, being dependent on the choice of origin (location of the interface). This issue has been considered in Ref. 6 and a certain prescription for choice of origin has been given. We prefer instead to define the interface to simply coincide with the midpoint of the interfacial cation-anion bond. That way, with the VBO and a knowledge of the electric fields, one can obtain the profile of the electrostatic potential everywhere in the heterostructure.

In Fig. 3, we show the electrostatic potential as well as the valence and conduction edges for the $(\mathrm{GaN})_{4} /(\mathrm{AlN})_{4}$ heterostructure. We find a slight asymmetry between the band offset for the Ga-face and $\mathrm{N}$-face interfaces, with the VBO being $1.15 \mathrm{eV}$ and $1.26 \mathrm{eV}$, respectively, a difference that is small but consistently found in our calculations. Figure 4 summarizes the calculated VBO as a function of the aluminum concentration.

There are a number of experiments on the measurement of the VBO for the GaN/AIN heterostructure. While the experiments agree on the type-I band alignment, the reported magnitudes vary widely, between $0.15 \mathrm{eV}$ (Ref. 7) and 1.4 $\mathrm{eV}$ (Refs. 8-10). The large spread could be the result of

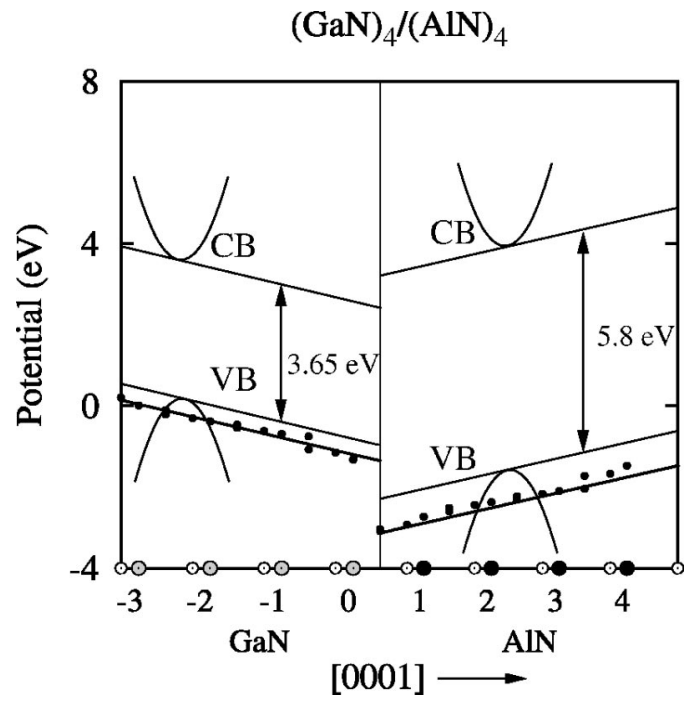

FIG. 3. Electrostatic potential for individual atoms using Eq. (2) and the positions of the valence and conduction bands near the interface. The vertical line indicates the position of the "Ga-face" interface. The "N-face" interface is located at the extreme right part of the figure, beyond the fourth AlN bilayer, at the midpoint between the last two atoms shown on the $x$ axis. The conduction edge for AlN has been reduced from its bulk value by a DFT-estimated $0.5 \mathrm{eV}$ to take into account the strain condition.

several factors such as uncontrolled strain effects or the difficulty of interpreting the photoemission spectra, which is the basis for many of the experimental results. For example, photoemission measurements for hexagonal AlN/GaN structures yield $0.60 \pm 0.24 \mathrm{eV}$ for $\mathrm{GaN}$ on $\mathrm{AlN}$, but it is not clear whether the interface is pseudomorphic. ${ }^{11}$ Earlier measurements by the same group for a thin GaN film grown on hexagonal AIN and vice versa yielded a VBO of $0.8 \pm 0.3$ $\mathrm{eV}^{12}$ Again, using the photoluminescence spectra for $\mathrm{GaN}: \mathrm{Fe}$ and $\mathrm{AlN}: \mathrm{Fe}$ using the $\mathrm{Fe}$ acceptor as the common reference level, Baur et al. ${ }^{13}$ obtained a value of $0.5 \mathrm{eV}$. The highest value reported so far was obtained by Sitar et al. ${ }^{8}$

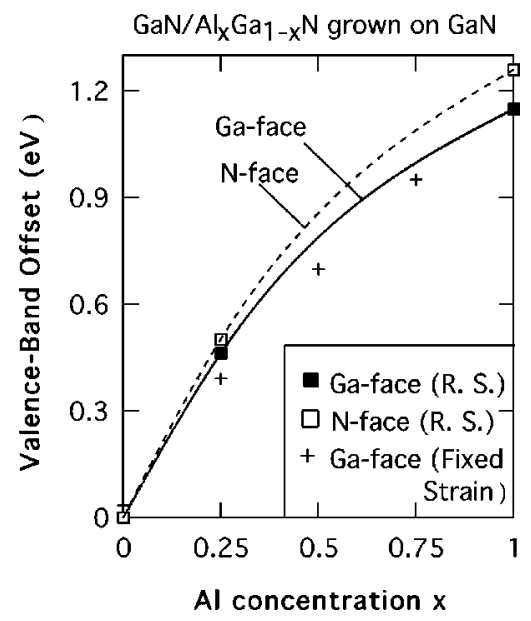

FIG. 4. Dependence of the VBO on the Al concentration. Strain for the $\mathrm{Al}_{x} \mathrm{Ga}_{1-x} \mathrm{~N}$ epilayer was relaxed (relaxed strain indicated by R. S. in the figure). Shown are also the results for the fixed strain Ga-face interface (the "+" data points), where strain was fixed, irrespective of $x$, to the value corresponding to AlN lattice matched to GaN. Lines indicate the interpolated results. 


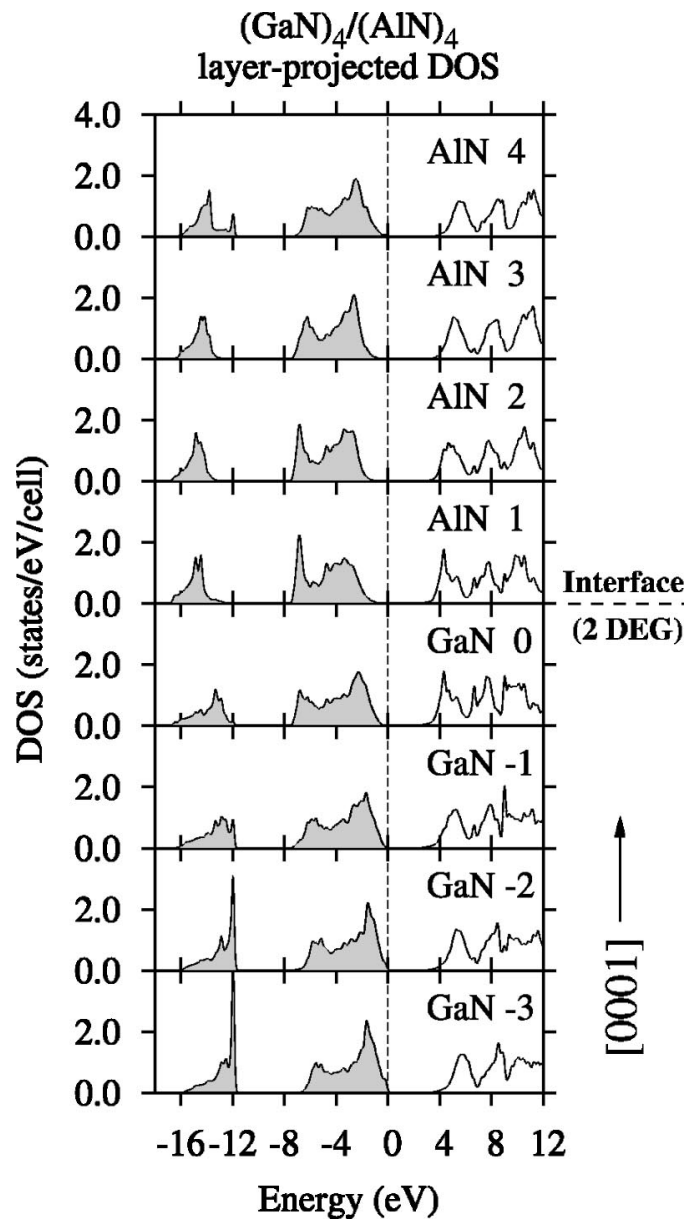

FIG. 5. Projected density of states for different bilayers (numbered consecutively beginning at the Ga-face interface). Notice that the conduction and valence band edges track the macroscopic electric field. The lowest conduction states for the entire heterostructure are at the Ga-face interface (indicated by the dashed line), so that electrons will form a 2DEG there, as is well known experimentally.

from cathodoluminescence data, which gave a value of 1.4 $\mathrm{eV}$ for the strained GaN/AIN heterostructures grown on $\mathrm{SiC}$. Concerning the doping dependence of the $\mathrm{VBO}$, there is a single measurement to our knowledge by Foxon et al. ${ }^{1}$ with the estimate of $0.2 \mathrm{eV}$ for $x=0.2$ and $\sim 1 \mathrm{eV}$ for $x=1$, in excellent agreement with our results. Concerning theory, much of the earlier theoretical studies treated a nonpolar interface, which is somewhat simpler because of the absence of the macroscopic electric fields, with the calculated VBO varying generally between 0.8 and $1.0 \mathrm{eV} .^{14,15}$ There are several calculations $\mathrm{s}^{4,6,16}$ for the polar GaN/AIN (0001) interface, all of which use the pseudopotential technique, yielding a VBO of about $0.70 \mathrm{eV}^{16}$

To discuss the electronic structure at the interface, it is convenient to show the layer-projected density of states (DOS) for the individual bilayers. The results, shown in Fig. 5 , confirm that there is no state in the gap induced by the interface. Also, the presence of the macroscopic electric field may be seen in the progressive shifting of the valence and conduction edges as one moves away from the interface. It is clear from the positions of the band edges in different bilayers that added electrons in the system (e.g., from donors or

\section{$\mathrm{GaN} / \mathrm{Al}_{x} \mathrm{Ga}_{1-\mathrm{x}} \mathrm{N}$ lattice-matched to $\mathrm{GaN}$}

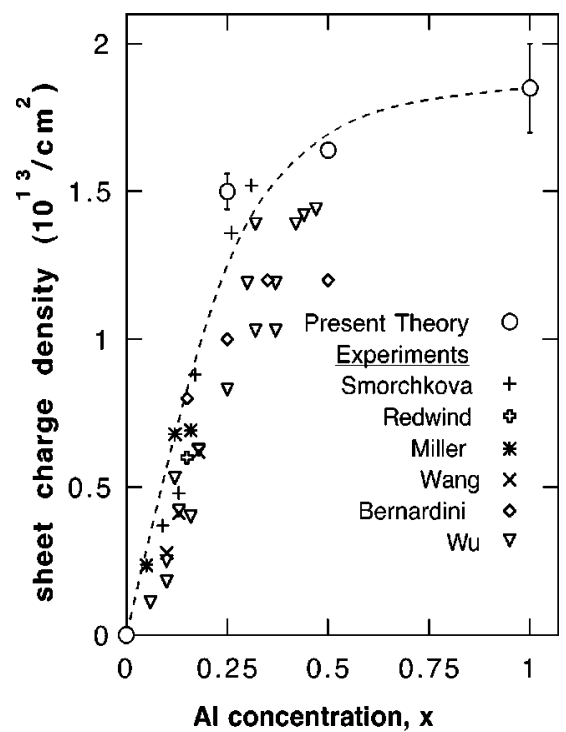

FIG. 6. DFT-calculated sheet charge density s as a function of the $\mathrm{Al}$ concentration (open circles) compared to the experimental data in the literature (see Refs. 17-22). The dashed line is a fit to the theory to guide the eye. The error bars in the theory data points are due to slight differences in the calculated electric fields, depending on how the planar-averaged potential (see Fig. 2, upper panel for $x=1$ ) was fitted to compute the electric fields.

from the surface states) will form a 2DEG at the Ga-face interface, while the holes will migrate to the $\mathrm{N}$-face interface (highest valence states are there) forming a two-dimensional hole gas. However, once the carriers (electrons or holes) migrate to the interface, the electric fields will be drastically reduced, extending the carriers to a distance of $100 \AA$ or more, which is much larger than what may be naively inferred from Fig. 5. To obtain the spatial extent of the resulting 2DEG from DFT, it is necessary to perform selfconsistent calculations with the added carriers, which we have not done here.

To calculate the polarization sheet charge density $\sigma$, we first compute the electric fields on either side of the interface and then use the result $E_{1}+E_{2}=\sigma / \epsilon_{0}$ from elementary electrostatics. We find that $\sigma$ first increases rapidly with $\mathrm{Al}$ concentration before saturating quickly to a value of about 2 $\times 10^{13} \mathrm{~cm}^{-2}$ (see Fig. 6). Interestingly, the inability to increase the carrier density beyond $x \approx 0.35$ has been noted earlier, ${ }^{23}$ but the authors had attributed it to reduced doping efficiency. Our results suggest that there is a more fundamental reason for this saturation-viz., the saturation of the electric fields near the interface with increasing concentration $x$, which in turn saturate the sheet charge density. Our calculated sheet charge density agrees quite well with the experimental data reported in the literature as well as with results obtained from macroscopic polarization theory. ${ }^{21,23,24}$

\section{ACKNOWLEDGMENTS}

S.S. would like to thank the National Research Council and the Air Force Office of Scientific Research for support of this work through a Summer Faculty Fellowship in the Air Force Research Laboratory, Dayton, Ohio. Z.S.P. would like 
to acknowledge support of this work by the U.S. Department of Energy through Grant No. DE-FG02-00ER45818.

${ }^{1}$ C. T. Foxon, S. V. Novikov, L. X. Zhao, and I. Harrison, Appl. Phys. Lett. 83, 1166 (2003).

${ }^{2}$ O. K. Andersen, Phys. Rev. B 12, 3060 (1975); O. K. Andersen and O. Jepsen, Phys. Rev. Lett. 53, 2571 (1984).

${ }^{3}$ L. D. Landau and E. M. Lifshitz, Theory of Elasticity, 3rd ed. (Pergamon, Oxford, 1986); B. Jogai, J. Appl. Phys. 91, 3721 (2002).

${ }^{4}$ M. B. Nardelli, K. Rapcewicz, and J. Bernholc, Phys. Rev. B 55, 7323 (1997).

${ }^{5}$ W. R. L. Lambrecht, B. Segall, and O. K. Andersen, Phys. Rev. B 41, 2813 (1990).

${ }^{6}$ F. Bernardini and V. Fiorentini, Phys. Rev. B 57, 9427 (1998).

${ }^{7}$ A. Rizzi, R. Lantier, F. Monti, H. Lüth, F. Della Sala, A. Di Carlo, and P. Lugli, J. Vac. Sci. Technol. B 17, 1674 (1999); R. Lantier, F. Bosherini, A. Rizzi, F. D’Acapito, S. Mobilio, and H. Lüth, Phys. Status Solidi A 176, 615 (1999).

${ }^{8}$ Z. Sitar, M. J. Paisley, B. Yan, F. Davis, J. Ruan, and J. W. Choyeke, Thin Solid Films 200, 311 (1991).

${ }^{9}$ J. R. Waldrop and R. W. Grant, Appl. Phys. Lett. 68, 2879 (1996).

${ }^{10}$ R. A. Beach, E. C. Piquette, R. W. Grant, and T. C. McGill, Mater. Res. Soc. Symp. Proc. 482, 775 (1998).

${ }^{11}$ G. Martin, A. Botchkarev, A. Rockett, and H. Morkoç, Appl. Phys. Lett. 68, 2541 (1996).

${ }^{12}$ G. Martin, S. Strite, A. Botchkarev, A. Agarwal, A. Rockett, H. Morkoç,
W. R. L. Lambrecht, and B. Segall, Appl. Phys. Lett. 65, 610 (1994).

${ }^{13}$ J. Baur, K. Maier, M. Kunzer, U. Kaufmann, and J. Schneider, Appl. Phys. Lett. 65, 2211 (1994).

${ }^{14}$ E. A. Albanesi, W. R. L. Lambrecht, and B. Segall, Mater. Res. Soc. Symp. Proc. 339, 607 (1994); J. Vac. Sci. Technol. B 12, 2470 (1994).

${ }^{15}$ S.-H. Wei and A. Zunger, Appl. Phys. Lett. 69, 2719 (1996); C. G. Van de Walle and J. Neugebauer, Appl. Phys. Lett. 70, 2577 (1997); J. A. Majewski and M. Staedele, Mater. Res. Soc. Symp. Proc. 482, 917 (1998).

${ }^{16}$ N. Binggelli, P. Ferrara, and A. Balderesci, Phys. Rev. B 63, 245306 (2001).

${ }^{17}$ I. P. Smorchkova et al., J. Appl. Phys. 86, 4520 (1999); Y. Zhang et al., ibid. 87, 7981 (2000).

${ }^{18}$ J. M. Redwing, M. A. Tischler, J. S. Flynn, S. Elhamri, M. Ahoujja, R. S. Newrock, and W. C. Mitchel, Appl. Phys. Lett. 69, 963 (1996).

${ }^{19}$ E. J. Miller, E. T. Yu, C. Poblenz, C. Elsass, and J. S. Speck, Appl. Phys. Lett. 80, 3551 (2002).

${ }^{20}$ T. Wang, Y. Ohno, M. Lachab, D. Nakagawa, T. Shirahama, S. Sakai, and H. Ohno, Appl. Phys. Lett. 74, 3531 (1999).

${ }^{21}$ V. Fiorentini, F. Bernardini, and O. Ambacher, Appl. Phys. Lett. 80, 1204 (2002).

${ }^{22}$ Y. F. Wu, B. P. Keller, P. Fini, S. Keller, T. J. Jenkins, L. T. Kehias, S. P. Denbaars, and U. K. Mishra, IEEE Electron Device Lett. 19, 50 (1998).

${ }^{23}$ M. S. Shur, A. D. Bykhovski, and R. Gaska, Solid-State Electron. 44, 205 (2000).

${ }^{24}$ O. Ambacher et al., J. Appl. Phys. 87, 334 (2000). 